KYUNGPOOK Math. J. 49(2009), 647-650

\title{
On the Iterated Duggal Transforms
}

\author{
MuneO CHO \\ Department of Mathematics, Kanagawa University, Yokohama 221-8686, Japan \\ e-mail : chiyom01@kanagawa-u.ac.jp \\ IL BONG JunG* \\ Department of Mathematics, Kyungpook National University, Daegu 702-701, Korea \\ e-mail : ibjung@knu.ac.kr
}

\author{
Woo Young LeE \\ Department of Mathematics, Seoul National University, Seoul 151-742, Korea \\ e-mail: wylee@math.snu.ac.kr
}

Abstract. For a bounded operator $T=U|T|$ (polar decomposition), we consider a transform $\widehat{T}=|T| U$ and discuss the convergence of iterated transform of $\widehat{T}$ under the strong operator topology. We prove that such iteration of quasiaffine hyponormal operator converges to a normal operator under the strong operator topology.

\section{Introduction}

Let $\mathcal{H}$ be a separable, infinite dimensional, complex Hilbert space and let $\mathcal{L}(\mathcal{H})$ be the algebra of all bounded linear operators on $\mathcal{H}$. An operator $T \in \mathcal{L}(\mathcal{H})$ has the unique polar decomposition $T=U|T|$, where $|T|=\left(T^{*} T\right)^{1 / 2}$ and $U$ is a partial isometry with initial space $(\operatorname{ran}|T|)^{-}$, the closure of the range of $|T|$, and final space $(\operatorname{ran} T)^{-}$, the closure of the range of $T$. The Aluthge transform $\widetilde{T}=|T|^{1 / 2} U|T|^{1 / 2}$, which was first studied in [1] and was applied to the study of the invariant subspace problem via the sequence $\left\{\widetilde{T}^{(n)}\right\}_{n \in \mathbb{N}}$ of Aluthge iterates of $T$, defined by $\widetilde{T}^{(1)}:=\widetilde{T}$ and $\widetilde{T}^{(n+1)}:=\left(\widetilde{T}^{(n)}\right)^{\sim}$ for $n \in \mathbb{N}$, in [8]. The problem whether Aluthge iteration of bounded operators on a Hilbert space $\mathcal{H}$ is convergent was introduced in [8]. If $\operatorname{dim} \mathcal{H}<\infty$, the Aluthge iteration converges to a normal operator (cf. [3], [2]). However, in general such iterations do not always converge under the strong operator topology (SOT) (cf. [4]). Moreover, the problem whether the hyponormal operators on $\mathcal{H}$ with $\operatorname{dim} \mathcal{H}=\infty$ has the Aluthge iteration converging to a normal operator in $\mathcal{L}(\mathcal{H})$ under the strong operator topology remains still open. In this paper we consider the transform $\widehat{T}=|T| U$, which is referred as the Duggal transform of $T$ in [5], and define $\widehat{T}^{(1)}:=\widehat{T}$ and $\widehat{T}^{(n+1)}:=\widehat{\left(\widehat{T}^{(n)}\right)}, n \in \mathbb{N}$, similarly.

* Corresponding author.

Received July 10, 2009; accepted August 21, 2009.

2000 Mathematics Subject Classification: 47B49, 47B20.

Key words and phrases: Aluthge transform, hyponormal operator, quasiaffinity. 
In this note we prove that if a quasiaffinity $T \in \mathcal{L}(\mathcal{H})$ is hyponormal, then the iteration of $\left\{\widehat{T}^{(n)}\right\}_{n \in \mathbb{N}}$ converges to a normal operator under SOT, whose spectrum is contained in the spectrum of $T$. In addition we give a sufficient condition with the iteration of $\left\{\widehat{T}^{(n)}\right\}_{n \in \mathbb{N}}$ for invariant subspaces for hyponormal operators in $\mathcal{L}(\mathcal{H})$.

\section{Duggal iterations}

We begin with some elementary properties, but sets forth basic relations between $T$ and $\widehat{T}$ that will be useful in the proofs below and the sequel research.

Basic properties (BP). Let $T=U|T|$ (polar decomposition) be in $\mathcal{L}(\mathcal{H})$. Then the following statements hold.

1) $|T| T=\widehat{T}|T|, U \widehat{T}=T U,|T|^{\frac{1}{2}} \widetilde{T}=\widehat{T}|T|^{\frac{1}{2}}$, and $\widetilde{T}\left(|T|^{\frac{1}{2}} U\right)=\left(|T|^{\frac{1}{2}} U\right) \widehat{T}$.

2) $T$ is a quasiaffinity if and only if $|T|$ is a quasiaffinity and $U$ is a unitary operator. And $\widehat{T}$ is a quasiaffinity if $T$ is. In this case, $T$ and $\widehat{T}$ are unitarily equivalent. Also, $\widehat{T}$ and $\widetilde{T}$ are quasisimilar.

3) The transform $\widehat{\imath}: \mathcal{L}(\mathcal{H}) \rightarrow \mathcal{L}(\mathcal{H})$ is SOT-continuous when restricted to the set of quasiaffinities.

4) $\sigma(T)=\sigma(\widehat{T})$, where $\sigma(\cdot)$ is the spectrum.

5) $\widehat{T}$ is invertible if and only if $\widetilde{T}$ is, and in this case, $\widehat{T}$ and $\widetilde{T}$ are similar.

The following theorem is contained in the main theorems of this paper.

Theorem 2.1. Suppose $T \in \mathcal{L}(\mathcal{H})$ is a quasiaffine hyponormal operator. Then the sequence $\left\{\widehat{T}^{(n)}\right\}_{n \in \mathbb{N}}$ converges in SOT to a normal operator $\widehat{T}_{L}$ such that $\sigma\left(\widehat{T}_{L}\right) \subset$ $\sigma(T),\left\|\widehat{T}_{L}\right\|=\|T\|$, or equivalently, $r\left(\widehat{T}_{L}\right)=r(T)$, where $r(\cdot)$ denotes the spectral radius.

Proof. Suppose $T=U|T|$ (polar decomposition), where $U$ is a unitary operator (via BP 2)). Then $|\widehat{T}|=\left(U^{*}|T|^{2} U\right)^{\frac{1}{2}}=U^{*}|T| U$, and so $\widehat{T}$ has the polar decomposition $\widehat{T}=U|\widehat{T}|$. Thus $\widehat{T}^{(2)}=|\widehat{T}| U=U^{*}|T| U^{2}$. By the mathematical induction we can see that

$$
\widehat{T}^{(n+1)}=U^{* n}|T| U^{n+1} \quad \text { for } n \in \mathbb{N}_{0}:=\mathbb{N} \cup\{0\} .
$$

On the other hand, if $T=U|T|$ is hyponormal then $|T|^{2}=T^{*} T \geq T T^{*}=U|T|^{2} U^{*}$, and hence $|T| \geq U|T| U^{*}$, or equivalently,

$$
U^{*}|T| U \geq|T| .
$$

From (2) we can see that $\left\{U^{* n}|T| U^{n}\right\}_{n \in \mathbb{N}}$ is a bounded increasing sequence of Hermitian operators. Therefore by the argument of [6, Prob. 120], $\left\{U^{* n}|T| U^{n}\right\}_{n \in \mathbb{N}}$ converges in SOT to a Hermitian operator $H$. Thus $\left\{\widehat{T}^{(n)}\right\}_{n \in \mathbb{N}}$ converges in SOT to $H U$, namely, $\widehat{T}_{L}$. In particular, since $U^{*} H U=H$ it follows that $H$ commutes with $U$ and therefore $\widehat{T}_{L}$ is normal. 
Towards the norm equality, since $H$ is the SOT-limit of $\left\{U^{* n}|T| U^{n}\right\}_{n \in \mathbb{N}}$, by (2) we have that

$$
U^{* n}|T| U^{n} \leq H \quad \text { for } n=0,1,2, \cdots
$$

and hence

$$
\||T|\|=\sup _{\|x\|=1}\langle|T| x, x\rangle \leq \sup _{\|x\|=1}\langle H x, x\rangle=\|H\| .
$$

Therefore

$$
\|T\|=\|U|T|\|=\||T|\| \leq\|H\|=\|H U\|=\left\|\widehat{T}_{L}\right\| .
$$

On the other hand, recall ([7, Cor. 3]) that if $S, S_{n} \in \mathcal{L}(\mathcal{H})$, for $n \in \mathbb{N}$, are hyponormal operators such that $S_{n}$ converges in SOT to $S$, then $\sigma_{a p}(S) \subseteq \liminf \sigma\left(S_{n}\right)$, where $\sigma_{a p}(\cdot)$ denotes the approximate point spectrum. Applying this result with $S_{n}=\widehat{T}^{(n)}$ and $S=\widehat{T}_{L}$, by BP 4 ) we have that

$$
\sigma_{a p}\left(\widehat{T}_{L}\right) \subseteq \sigma(T) \quad \text { and so } \quad \sigma\left(\widehat{T}_{L}\right) \subset \sigma(T) .
$$

Thus $r\left(\widehat{T}_{L}\right) \leq r(T)$. However since every hyponormal operator is normaloid, i.e., norm equals spectral radius, it follows that $\left\|\widehat{T}_{L}\right\| \leq\|T\|$. Therefore we can conclude that $\left\|\widehat{T}_{L}\right\|=\|T\|$, or equivalently, $r\left(\widehat{T}_{L}\right)=r(T)$.

In Theorem 2.1, the sequence $\left\{\widehat{T}^{(n)}\right\}$ does not always converge in SOT without the condition of hyponormality (see Example 2.2 below).

Example 2.2. Let $P$ be a positive quasiaffinity on $\mathcal{H}$ with $2 \leq \operatorname{dim} \mathcal{H} \leq \infty$ and let $U \in \mathcal{L}(\mathcal{H})$ be unitary such that $|T| U \neq U^{*}|T|$ and $U^{2}=1$. Consider an operator $T=U P$. Then, by BP 2) $T$ is quasiaffinity. Recall from (1) that $\widehat{T}^{(n+1)}=U^{* n}|T| U^{n+1}\left(n \in \mathbb{N}_{0}\right)$, which implies that

$$
\widehat{T}^{(n)}= \begin{cases}U^{*}|T| & \text { if } n \text { is odd } \\ |T| U & \text { if } n \text { is even. }\end{cases}
$$

Hence $\left\{\widehat{T}^{(n)}\right\}_{n \in \mathbb{N}}$ does not converge under SOT in $\mathcal{L}(\mathcal{H})$.

In Theorem 2.1 the property $\left\|\widehat{T}_{L}\right\|=\|T\|$ does not hold in general (see Proposition 2.3 below).

Proposition 2.3. Let $T \in \mathcal{L}(\mathcal{H})$ be a nilpotent operator of order $m \geq 2$. Then $(\widehat{T})^{m-1}=0$ and $\widehat{T}^{(m-1)}=0$. Therefore $\widehat{T}_{L}=0$.

Proof. It follows from [9, Prop. 4.6] that $(\widetilde{T})^{m-1}=0$. Since $(\widehat{T})^{m-1}|T|^{1 / 2}=$ $|T|^{1 / 2}(\widetilde{T})^{m-1}=0,(\widehat{T})^{m-1}=0$ on the space $(\operatorname{ran}|T|)^{-}$. And also, for $h \in$ $\left((\operatorname{ran}|T|)^{-}\right)^{\perp}$, obviously $(\widehat{T})^{m-1} h=0$. To prove the second part of this proposition, we will claim from the mathematical induction that if $T^{k}=0$, then $\widehat{T}^{(k-1)}=0$. For $k=2$, if $T^{2}=0$, then $\widetilde{T}=0$ (because, $|T| U|T|=0$, which implies from [9, Lem. 4.5] that $|T|^{1 / 2} U|T|^{1 / 2}=0$ ) and so, by BP 1$)$, we have $\widehat{T}|T|^{1 / 2}=|T|^{1 / 2} \widetilde{T}=0$. Hence it is easy to see that $\widehat{T}=0$. Assume that the assertion holds for $2 \leq k \leq m-1$. 
Suppose $T^{m}=0$. Then, since $(\widehat{T})^{m-1}=0$, by the induction hypothesis, we have $\widehat{(\widehat{T})}^{(m-2)}=0$, i.e., $\widehat{T}^{(m-1)}=0$. Hence the proof is complete.

Remark 2.4. We don't know whether every hyponormal operator $T$ in $\mathcal{L}(\mathcal{H})$ always has the Duggal iteration convergence $\widehat{T}_{L}$ under SOT.

Acknowledgment. The first author was supported by Kakenhi No. 20540192 and the second author was supported by Kyungpook National University Research Fund, 2008 .

\section{References}

[1] A. Aluthge, On p-hyponormal operators for $0<p<1$, Integral Equations Operator Theory, 13(1990), 307-315.

[2] T. Ando and T. Yamazaki, The iterated Aluthge transforms of a 2-by-2 matrix converge, Linear Algebra Appl., 375(2003), 299-309.

[3] J. Antezana, E. Pujals, and D. Stojanoff, Convergence of the iterated Aluthge transform sequence for diagonalizable matrices, Advances Math., 216(2007), 255-278.

[4] M. Cho, I. Jung, and W. Lee, On Aluthge transforms of p-hyponormal operators, Integral Equations Operator Theory, 53(2005), 321-329.

[5] C. Foias, I. Jung, E. Ko, and C. Pearcy, Complete contractivity of maps associated with the Aluthge and Duggal transforms, Pacific J. Math., 209(2003), 249-359.

[6] P. R. Halmos, A Hilbert space problem book, 2nd ed., New York, 1982.

[7] I. S. Hwang and W. Y. Lee, The spectrum is continuous on the set of p-hyponormal operators, Math. Z., 235(2000), 151-157.

[8] I. Jung, E. Ko, and C. Pearcy, Aluthge transforms of operators, Integral Equations Operator Theory, 37(2000), 437-448.

[9] I. Jung, E. Ko, and C. Pearcy, The iterated Aluthge transform of an operator, Integral Equations Operator Theory, 45(2003), 375-387. 